\title{
Caractéristiques des peuplements ligneux du tracé de la grande muraille verte des provinces de Wadi-Fira Est et de l'Ennedi Est du Tchad
}

\author{
Mékila MBAIKOUBOU ${ }^{1,2^{*}}$, Abakar GUIHINI ${ }^{1}$, Aly DIALLO $^{1}$, Ndiabou FAYE $^{1}$, \\ Minda MAHAMAT Saleh ${ }^{1}$, Tahir Doudja MOUSSA ${ }^{2}$ et Aliou GUISSE ${ }^{1,3^{*}}$
}

\author{
${ }^{1}$ Département Biologie Végétale, Faculté des Sciences et Techniques, Université Cheikh Anta Diop de Dakar, \\ B.P 5005 Dakar, Sénégal. \\ ${ }^{2}$ Institut Tchadien de Recherche Agronomique pour le Développement (ITRAD), BP : 5400 N'Djaména, Tchad. \\ ${ }^{3}$ Observatoire Homme Milieu Tessekere, UMI 3189, Tessékéré, Sénégal. \\ *Auteur correspondant ; E-mail: mekilam@yahoo.fr ; Tel. (+235) $66491269 / 92540194$
}

\begin{tabular}{lll}
\hline Received: 13-07-2021 & Accepted: 30-09-2021 & Published: 30-10-2021 \\
\hline
\end{tabular}

\section{RESUME}

Pour faire des propositions de stratégies de gestion durable de la grande muraille verte, la connaissance des caractéristiques du peuplement ligneux du tracé du Tchad est capitale. L'étude a pour objet de caractériser la flore et la végétation ligneuse sur le tracé de la GMV du Tchad, précisément au Wadi Fira Est et à l'Ennedi Est. La méthodologie utilisée a consisté à déterminer la composition floristique et la structure du peuplement ligneux dans les deux sites. Les inventaires floristiques ont permis de recenser 8 espèces reparties en 7 genres et 4 familles dans le site de Wadi Fira Est tandis que dans le site de l'Ennedi Est nous avons recensé 7 espèces reparties en 6 genres et 4 familles. La flore ligneuse est dominée par les Mimosaceae. La densité des ligneux (39,43 ind.ha $\left.{ }^{-1}\right)$ est plus importante dans l'Ennedi Est que dans le Wadi Fira Est (36,62 ind.ha $\left.{ }^{-1}\right)$; Cependant, le recouvrement au Wafi Fira Est $\left(559,428 \mathrm{~m}^{2} \cdot \mathrm{ha}^{-1}\right)$ est plus important que celui de l'Ennedi Est $\left(499,844 \mathrm{~m}^{2} \cdot \mathrm{ha}^{-1}\right)$. Les résultats obtenus montrent que les espèces les plus adaptées aux conditions écologiques pour la réussite de la GMV sont Acacia raddiana et Balanites aegyptiaca qui conviennent pour la restauration de ces écosystèmes.

(C) 2021 International Formulae Group. All rights reserved.

Mots clés : Tchad, Muraille verte, végétation, peuplement, régénération.

\section{Characteristics of the woody stands of the route of the great green wall of the provinces of Wadi-Fira East and Ennedi East of Tchad}

\begin{abstract}
To make proposals for sustainable management strategies for the Great Green Wall, knowledge of the characteristics of the woody population along the Chad route is essential. The purpose of the study is to characterize the flora and woody vegetation along the route of the GGW of Chad, specifically in Wadi Fira East and Ennedi East. The methodology used consisted in determining the floristic composition and the structure of the woody stand in the two sites. Floristic inventories made it possible to identify 8 species divided into 7 genera and 4 families in the Wadi Fira East site while in the Ennedi East site we have identified 7 species divided into 6 genera and 4 families. The woody flora is dominated by Mimosaceae. The density of woody plants (39.43
\end{abstract}


ind.ha ${ }^{-1}$ ) is higher in Ennedi East than in Wadi Fira East (36.62 ind.ha-1); However, the overlap at Wafi Fira East $\left(559.428 \mathrm{~m}^{2} \cdot \mathrm{ha}^{-1}\right)$ is greater than that at Ennedi East $\left(499.844 \mathrm{~m}^{2} \cdot \mathrm{ha}^{-1}\right)$. The results obtained show that the species most suited to ecological conditions for the success of GMV are Acacia raddiana and Balanites aegyptiaca which are suitable for the restoration of these ecosystems.

(C) 2021 International Formulae Group. All rights reserved.

Keywords: Tchad, Green wall, vegetation, population, regeneration.

\section{INTRODUCTION}

Au Sahel et particulièrement au Tchad, les espèces ligneuses jouent un rôle prépondérant dans la vie socio-économique et culturelle des populations. En effet, les ligneux rentrent dans le cycle bio-géochimique des systèmes de production par apport de la biomasse et/ou de la nécromasse, la création de microclimat favorable aux cultures et la protection des sols contre les érosions hydrique et éolienne (Ba et al., 2018). De plus, les ligneux constituent des apports importants en alimentations humaine et animale surtout pendant les périodes difficiles de l'année au Sahel (Moussa et al., 2015). L'importance des végétaux est reconnue dans beaucoup de services qu'ils rendent à la population humaine (Kaou et al., 2017) ; ils constituent des sources alimentaires essentielles pour les animaux (Sarr et al., 2013). Ils créent un microclimat qui favorise les cultures et protège les sols contre les érosions (Ngom et al., 2018). En outre, ils sont une source principale d'énergie et procurent du bois d'usage domestique, du bois d'œuvre et des produits de la pharmacopée traditionnelle pour les populations rurales (Larwanou et al., 2006 ; Dan Guimbo et al., 2010 ; Laouali et al., 2014). La strate ligneuse a particulièrement été touchée à cause d'une demande croissante des populations en bois de feu, fourrage et produits divers. L'une des manifestations immédiates de cette dégradation est la réduction de la densité de la végétation (Minda et al., 2015) et de la biodiversité végétale, imputables respectivement aux conditions climatiques drastiques et à l'action anthropique. Cependant, cette tendance de la dégradation des ressources naturelles ne serait pas irréversible si l'activité de l'homme était rationalisée.
L'anthropisation du peuplement ligneux est un aspect très important pour comprendre l'évolution des rapports entre les sociétés humaines et leur environnement (Jalut et al., 2000 ; Salvador et al., 2002 ;Van Geel et Magny, 2002). En effet, l'action de l'homme sur l'environnement concerne les activités agro-pastorales notamment l'exploitation forestière qui a pour conséquence des modifications plus ou moins intenses de la structure des formations végétales (Magrin et Martin, 2004).

Pour faire face à cette situation, les pays du Sahel ont initié un grand projet dénommé Grande Muraille Verte (GMV), une stratégie pour lutter contre la désertification et réhabiliter les sols dégradés. Cette GMV est une ceinture de végétale de plusieurs espèces, sur une longueur d'environ $7000 \mathrm{~km}$ et une largeur de $15 \mathrm{~km}$ et qui englobe onze pays africains (Sénégal, Burkina Faso, Djibouti, Erythree, Ethiopie, Mali, Mauritanie, Niger, Nigeria, Soudan et Tchad) sur l'axe DakarDjibouti (Grande Muraille Verte, 2009), (Figure 1).Cette nouvelle dynamique, nécessite la réactualisation des connaissances sur la végétation notamment la végétation ligneuse des parcs agroforestiers en vue d'appréhender la structure, la richesse floristique et la capacité de régénération des peuplements ligneux pour faciliter la gestion de ces ressources au profit des populations. Cette étude s'inscrit dans une telle dynamique afin de contribuer à caractériser les peuplements ligneux. La connaissance des caractéristiques du peuplement végétal permet de mieux comprendre les écosystèmes de cette zone, de les décrire dans leurs aspects afin de proposer des stratégies de gestion durable.

L'objectif général de cette étude est de contribuer à l'amélioration de la productivité 
des ligneux pour une conservation durable des ressources naturelles face aux effets du changement climatique au Tchad. Plus spécifiquement, il s'agit de caractériser les différentes espèces ligneuses (diversité, floristique, densité, recouvrement, surface terrière et régénération naturelle) et leurs potentialités dans les provinces de Wadi Fira Est et de l'Ennedi Est sur le tracé de la grande muraille verte.

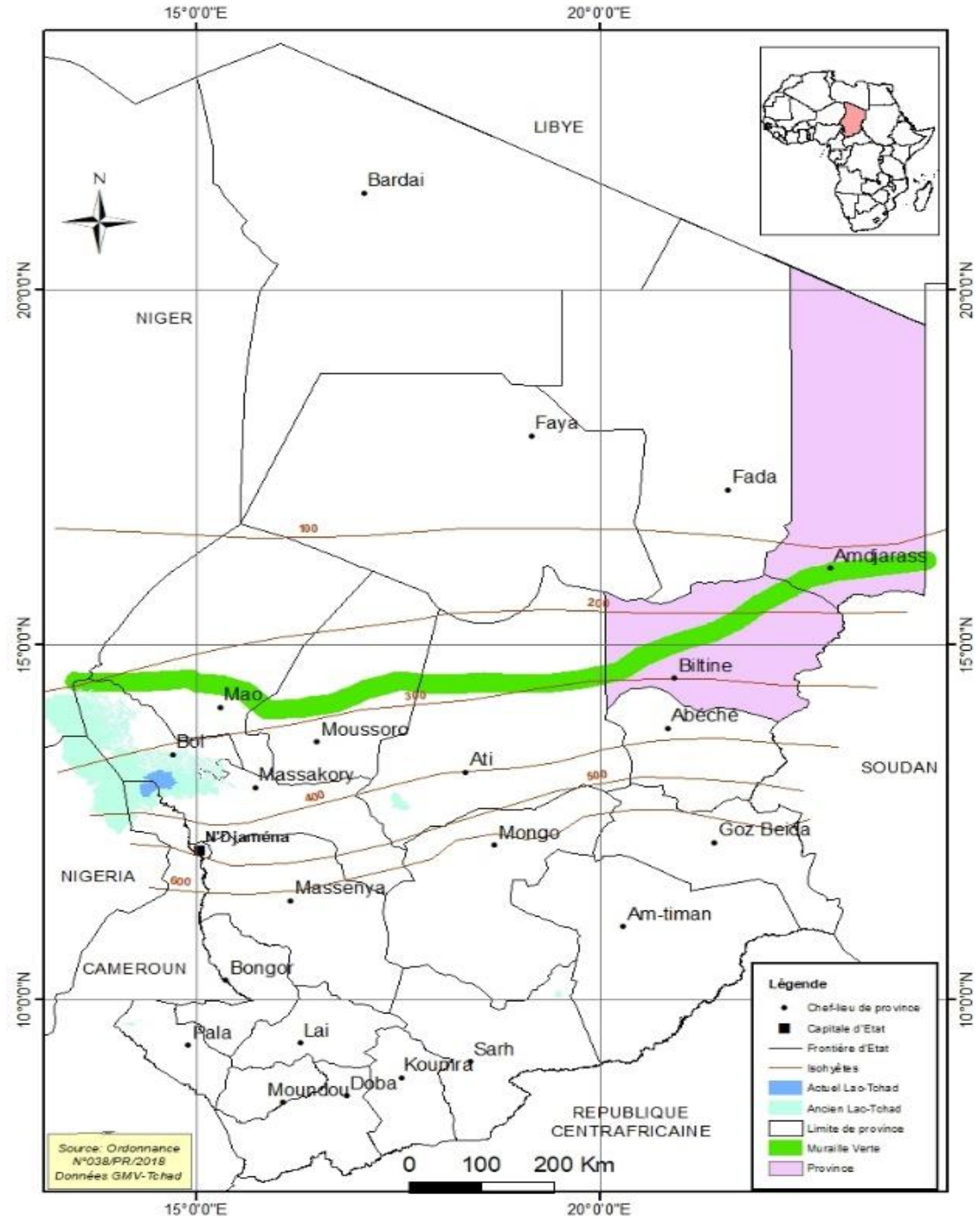

Figure 1 : Tracé de la grande muraille verte (Source : CNRD, 2020). 


\section{MATERIEL ET METHODES}

\section{La zone d'étude}

La zone d'étude s'étend sur $277 \mathrm{~km}$ de long et $15 \mathrm{~km}$ de large, elle fait partie du tracé de la GMV du Tchad (Figure 2). Le climat est de type sahélien caractérisé par deux saisons : une saison sèche qui s'étend de novembre à mai et une saison pluvieuse de juin à octobre. La température varie de $22,31{ }^{\circ} \mathrm{C}$ à $33,64{ }^{\circ} \mathrm{C}$ (ASECNA, 2012). Deux sites (Wadi Fira Est et Ennedi Est) ont fait l'objet de l'étude pour la caractérisation des ligneux de la zone d'étude. Leur choix a été motivé par les différentes unités pédologiques rencontrées, les changements de végétations observés et les types de sol.

Les provinces du Wadi Fira Est et de l'Ennedi Est sont caractérisées par un climat de type sahélo saharien avec une faible pluviométrie de trois mois qui débute en juillet et finit à la fin de septembre. La pluviométrie moyenne annuelle est de $250 \mathrm{~mm}$ dans la Province de Wadi Fira Est au Sud et $100 \mathrm{~mm}$ à Fada au Nord de la Zone. Le mois d'août est le seul mois le plus pluvieux dans la zone. (ANAM, 2020). L'amplitude thermique varie entre $26^{\circ} \mathrm{C}$ à $34^{\circ} \mathrm{C}$. Les mois les plus chauds sont les mois de mars, avril, mai et juin. La température maximale est observée au mois de mai avec une moyenne de $41,7^{\circ} \mathrm{C}$ et la température minimale est observée au mois de janvier et décembre. La température moyenne au mois d'août est de $26^{\circ} \mathrm{C}$ (Figure 3) (ANAM, 2020).

\section{Evolution du climat dans la zone d'étude}

L'analyse des isohyètes de 1950 à 2019 place la zone entre l'isohyète $100 \mathrm{~mm}$ au Nord et $300 \mathrm{~mm}$ au Sud. L'évolution de ces isohyètes montre une diminution de la pluviométrie de 10\% du Nord vers le Sud de 1950 à 1990 avec un glissement d'environ $90 \mathrm{~km}$ et une augmentation de la pluviométrie de $12 \%$ est observée dans la zone depuis la normale de 1991 à 2010. (Figure 4). (ANAM, 2020).

\section{Collecte des données}

L'échantillonnage a été effectué selon la méthode de Niang et al. (2014) à partir de 27 placettes de $50 \mathrm{~m}$ x $50 \mathrm{~m}\left(2500 \mathrm{~m}^{2}\right)$ de surface chacune dans chaque site en fonction des différentes unités morphopédologiques (dune, plateau et dépression), de la variation de la végétation et des changements observés au niveau de la nature des sols.

Dans chaque placette, la liste des espèces a été établie. Des mesures dendrométriques ont été effectuées. Les mensurations ont concerné les individus à circonférence basale supérieure ou égale à 10 cm (Minda et al., 2013). Pour chaque individu rencontré, les mesures ont porté sur la circonférence à la base du tronc à $30 \mathrm{~cm}$ du sol, le diamètre de la projection du houppier au sol dans deux directions (Nord-Sud et Est-Ouest), la hauteur des arbres et la distance entre deux arbres. Ces mesures ont été cumulées au comptage de la régénération du peuplement. L'inventaire et l'analyse floristique ont permis de dresser la liste des espèces recensées et de les regrouper par famille.

Les paramètres suivants ont été déterminés :

- La densité réelle qui est le nombre d'individus par unité de surface (individus.ha1) ; (Jauffret, 2001 ; Minda et al., 2013) a été calculée par la formule suivante :

Densité réelle (individu.ha ${ }^{-1}$ ) $=$ Nombre $\mathrm{d}$ 'individus de l'espèce/Surface étudiée en ha $\mathrm{x}$ 100.

- La densité théorique qui peut être exprimée par l'écartement moyen entre les individus en utilisant la méthode du plus proche individu. Son calcul se fait par le rapport de la surface d'un hectare $\left(10000 \mathrm{~m}^{2}\right)$ sur le carré de la distance moyenne entre les arbres ( $\mathrm{dm}$ ) (Minda et al., 2015) selon la formule suivante :

Densité théorique (individus.ha' ${ }^{-1}$ ) $=10$ 000/ $(\text { dmoy })^{2}$

$\mathrm{dm}:$ représente la distance moyenne entre les individus (m) ; 
- La surface terrière (St) ou recouvrement basal, est la somme des surfaces terrières de tous les individus dont la circonférence basale (C) est supérieure ou égale à $10 \mathrm{~cm}$. Cette surface terrière peut être estimée en considérant que les sections des tiges sont circulaires. Elle s'exprime par unité de surface $\left(\mathrm{m}^{2} \cdot \mathrm{ha}^{-1}\right)$ et se calcule tel que décrit par Ndiaye et al., (2014) par la formule ci-après :

St $=\sum \mathbf{C}^{2} / \mathbf{4} \pi$

$\mathrm{St}=$ surface terrière exprimée en $\mathrm{m}^{2} \cdot \mathrm{ha}^{-1} ; \mathrm{C}=$ circonférence à $30 \mathrm{~cm}$ du sol des individus mesurée en centimètre.

- Le recouvrement aérien $(\mathrm{Sc})$ est la somme des surfaces de la couronne de tous les individus du peuplement. Elle est obtenue à l'aide du diamètre moyen du feuillage de l'arbre (D) assimilé à un cercle par projection sur le sol. Elle est obtenue par la formule suivante (Minda et al., 2015) :

$\mathrm{Sc}=\mathrm{St}=\sum \pi \cdot \mathrm{D}^{2} / 4$

$\mathrm{SC}=$ surface de la couronne exprimée en $\mathrm{m}^{2}$.ha-1; $\mathrm{D}=$ moyenne des diamètres Est/Ouest et Nord/Sud exprimée en mètre.

- Le taux de régénération du peuplement est donné par le rapport (en pourcentage) entre l'effectif total des jeunes plants (circonférence $<10 \mathrm{~cm}$ ) et l'effectif total du peuplement (Minda et al., 2013).

\section{TRP $=($ Effectif total des jeunes} plants/Effectif total du peuplement) x 100

Dans cette étude, le taux d'anthropisation du peuplement ligneux est évalué à partir du rapport exprimé en pourcentage du nombre de pieds exploités divisés par le nombre total d'individus par sites.

AP $=($ Nombre de pieds exploités/Nombre total d'individus) $x \mathbf{1 0 0}$
Indice de Shannon (H'), définition et formule

L'Indice de Shannon, également connu sous le nom de Shannon-Weaver, est utilisé pour quantifier une biodiversité spécifique. Le symbole H' est utilisé pour le représenter, et ses valeurs sont comprises entre des nombres positifs, généralement entre 2, 3 et 4 . Cet indice est l'un des plus populaires pour mesurer la biodiversité. Il est calculé par cette formule :

$\mathrm{H}=-\sum$ pi $\log 2$ pi

\section{Indice d'équitabilité de Pielou (E)}

L'équitabilité de Pielou ou régularité est une mesure du degré de diversité atteint par le peuplement et correspond au rapport entre la diversité effective $(\mathrm{H})$ et la diversité maximale théorique (Hmax) qui est égale au log à base 2 du nombre de taxons. Elle est ainsi déterminée à partir de les formules suivantes :

$\mathrm{E}=\mathrm{H} / \mathrm{H} \max$ ou $E=H / \log 2 S$

$S$ représente la richesse spécifique et $H$ l'indice de diversité de Shannon

L'équitabilité varie entre 0 et 1 . Elle tend vers 0 si la quasi-totalité des effectifs correspond à une seule espèce du peuplement et tend vers 1 lorsque chacune des espèces est presque représentée par le même nombre d'individus ou le même recouvrement. L'équitabilité de Pielou élevé peut être alors le signe d'un peuplement équilibré (Yaya et al., 2019).

\section{- Traitements des données}

Les données recueillies ont été gérés et traitées avec le tableur Excel et le logiciel Mintab afin d'évaluer les paramètres de structure. 


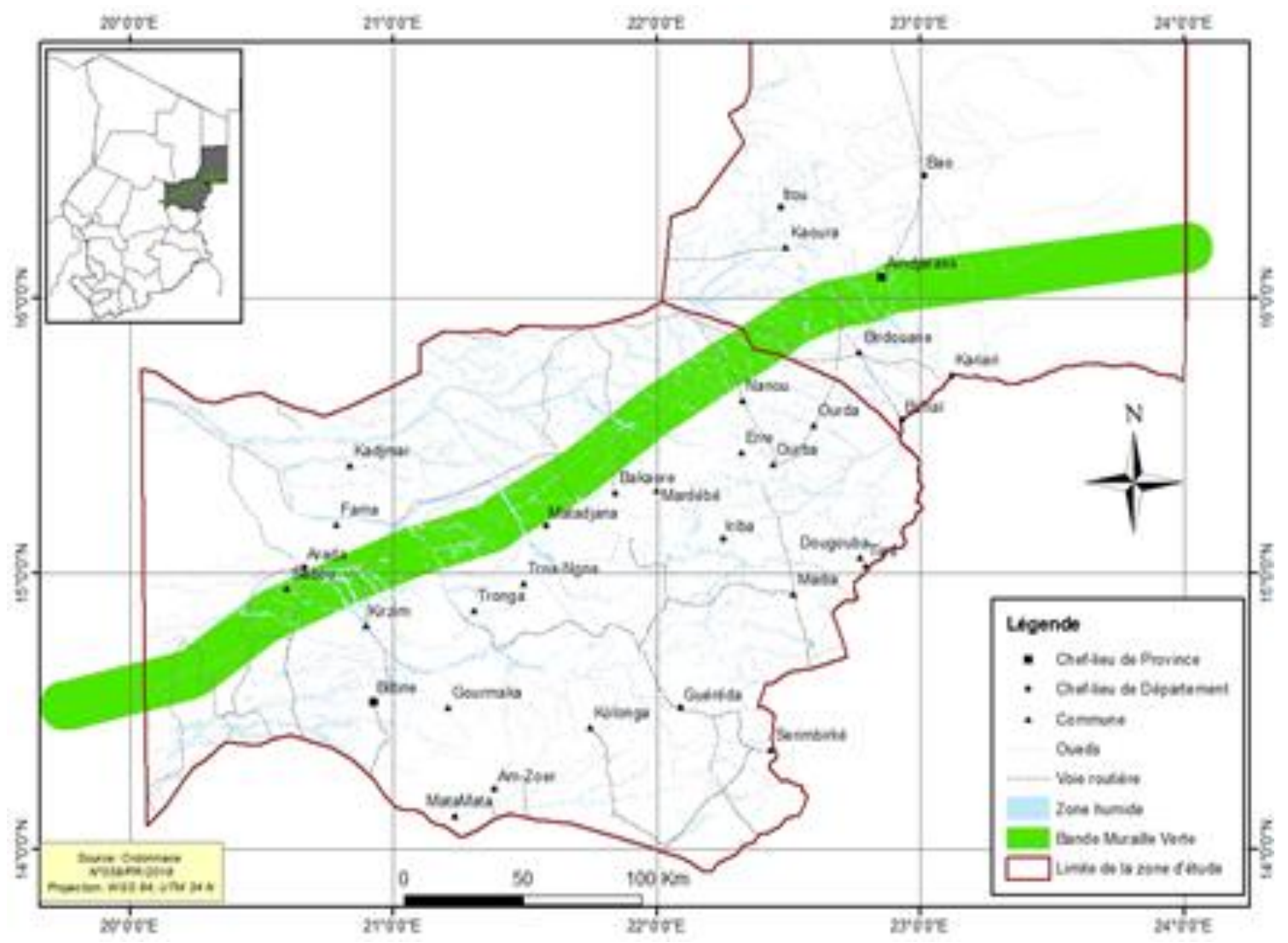

Figure 2 : Zone de l'étude (Source : CNRD, 2020).

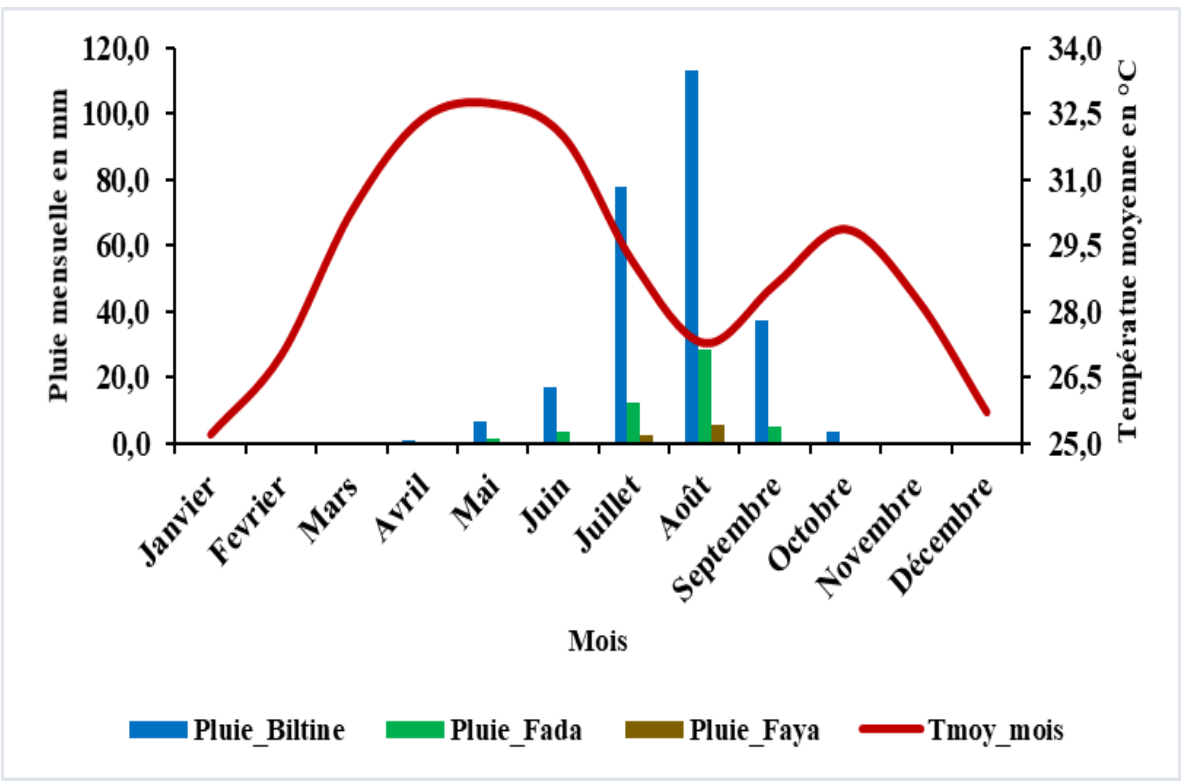

Figure 3 : Diagramme ombrothermique de la zone d'étude (Source : ANAM, 2020, Réalisation : Singambaye D). 


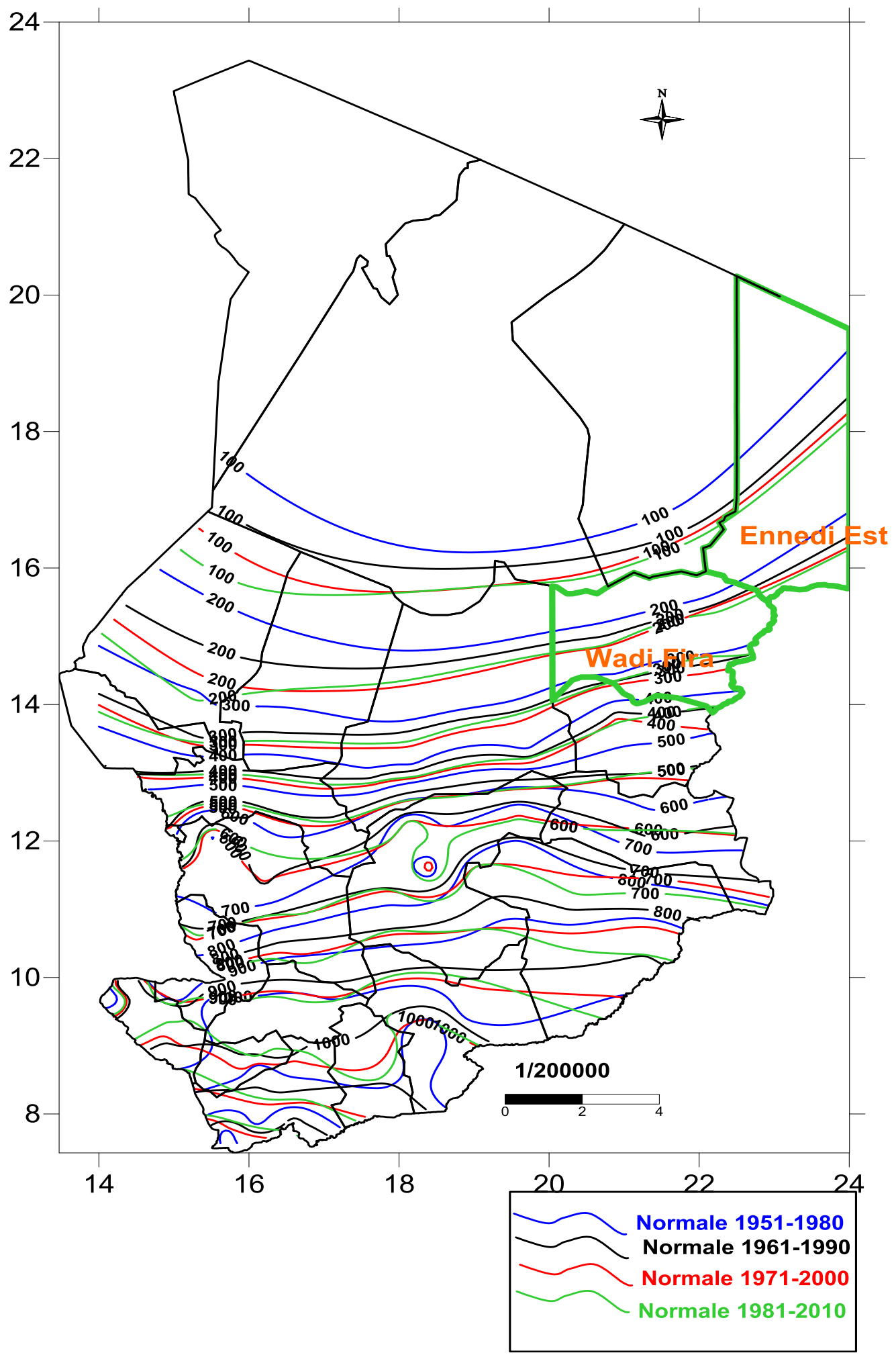

Figure 4: Evolution des isohyètes dans la zone d'étude (Source: ANAM, 2020, réalisation: Singambaye D). 


\section{RESULTATS}

\section{Composition et diversité floristique Liste floristique}

La flore ligneuse inventoriée dans la zone d'étude est riche de 8 espèces, réparties en 6 genres appartenant à 4 familles (Tableau.1). Les familles des Capparidacées ( 3 espèces) et des Mimosacées (3 espèces) sont les mieux représentées. Les autres familles sont représentées par une seule espèce. Prenant en compte le nombre d'espèces, de prime à bord le site de Wadi Fira Est présente la plus grande diversité floristique avec 08 espèces appartenant à 07 genres et 04 familles tandis le site de l'Ennedi Est présente 07 espèces appartenant à 06 genres et 04 familles. Toutes les espèces ont été trouvées sur les deux sites excepté l'espèce Faidherbia albida (Del.) A. Chev qui se trouve seulement dans le site de Wadi Fira Est.

\section{Fréquence des espèces en fonction des sites}

Dans le site de Wadi Fira Est, les différentes espèces ont été représentées par les fréquences suivantes: Acacia raddiana (Savi) Brenan (33,6\%), Balanites aegyptiaca (L.) Del (25,2\%), Boscia senegalensis (Pers.) $(15,1 \%)$, Zizyphus mauritiana Lam. (10,9\%), Capparis decidua (Forssk) Edgew (6,7\%), Acacia senegal (L,) Willd (5,9\%), Faidherbia albida (Del.) A. Chev. (1,7\%) et Maerua crassifolia Forssk $(0,8 \%)$. Pour le site de l'Ennedi Est les proportions des espèces par rapport au total inventorié étaient les suivantes: Acacia raddiana $(41,3 \%)$, M. crassifolia $(19,6 \%)$, Boscia senegalensis $(18,1 \%)$, A. senegal $(8 \%)$, C. decidua $(6,57 \%)$, Balanites aegyptiaca $(5,8 \%)$ et Ziziphus mauritiana $(0,7 \%)$.

Les espèces Acacia raddiana et Balanites aegyptiaca apparaissent comme les espèces les plus abondantes de la zone d'étude. Ces deux espèces appartenant respectivement à la famille des Mimosaceae et des Balanitaceae ont permis de définir le peuplement de la zone d'étude comme une végétation des Mimosaceae et des Balanitaceae. Certaines espèces par contre sont moins abondantes d'un site à l'autre. Il s'agit de Zizyphus mauritiana $10,9 \%$ à Wadi Fira Est et $0,7 \%$ à l'Ennedi Est et Maerua crassifolia ayant respectivement les fréquences de $0,8 \%$ et $19,6 \%$ sur les sites (Figure.5).

D'une manière générale, pour la zone de la présente étude, l'espèce dominante est Acacia raddiana car elle représente 38,97\% de la population inventoriée suivie de Boscia senegalensis $(17,71 \%)$ et de Balanites aegyptiaca (14,96\%). L'espèce Faidherbia albida était la moins représentée dans la zone d'étude avec $0,39 \%$.

\section{Diversité spécifique}

En considérant les zones, l'indice de Shannon $\left(\mathrm{H}^{\prime}\right)$ est plus élevé dans la zone d'Ennedi Est $(2,71)$ que dans la zone de Wadi Fira Est (Tableau. 2). Ces indices prouvent que même si la diversité floristique n'est pas assez forte dans ces zones, les espèces présentes dans chaque site sont distribuées de manière homogène.

L'indice d'équitabilité de Pielou (E) suit la même tendance que celui de Shannon

\section{Caractéristiques structurales des ligneux}

Sur l'ensemble de la zone étudiée, 257 individus ont été recensés soit une densité de 73,18 individus à l'hectare. Ces individus sont répartis dans les différentes unités (sites) de la manière suivante : Wadi Fira Est (119 individus) et Ennedi Est (138 individus) (Tableau.3).

La structure du peuplement ligneux dans les zones est présentée dans le tableau 3.

\section{Densité réelle et théorique}

Dans la zone d'étude, la densité réelle était de 38,08 pieds/hectare; Cependant, cette densité était plus élevée dans l'Ennedi Est $(39,43 \pm 5,4)$ que dans le Wadi Fira Est $(36,62$ $\pm 4,24)$.

Les distances moyennes entre deux arbres sont relativement élevées. Elles sont de l'ordre de 10,73 m dans le Wadi Fira Est à 12,52 m dans l'Ennedi Est.

Partout, la densité réelle est plus faible que la densité théorique. En effet, le rapport entre la densité théorique et la densité réelle est de 2,37 dans le Wadi Fira Est et de 1,62 dans l'Ennedi Est. Les espèces présentant les plus fortes densités dans les différents sites étaient Acacia raddiana et Balanites aegyptiaca avec des densités respectives de 12,31 et 9,23 ind.ha $^{-1}$ 
dans le Wadi fira Est ainsi qu'Acacia raddiana, Maerua crassifolia et Boscia senegalensis avec comme densités respectives $16,29,7,71$ et 7,14 ind.ha ${ }^{-1}$ dans l'Ennedi Est.

\section{Recouvrement}

En fonction des sites, le recouvrement aérien des ligneux varie et dépend de la présence de grands arbres aux larges houppiers. Pour le peuplement, il est de $17,82 \%$. Pour le site de Wafi Fira Est, il est de 18,18\% alors que pour l'Ennedi Est, il est de 17,79. La distribution des espèces par site se présente de la manière suivante :

A Wadi fira Est les espèces présentant un recouvrement important sont: Acacia raddiana (8,40\%), Balanites aegyptiaca (4,63\%), Faidherbia albida (1,73\%), Acacia senegal (1,43\%), Zizyphus mauritiana $(1,07 \%)$, Boscia senegalensis $(0,51 \%)$ et Capparis decidua $(0,36 \%)$. Maerua crassifolia présente un recouvrement faible et moins important $(0,05 \%)$.

Pour le site de l'Ennedi Est, ce sont les espèces Acacia raddiana (12,65\%), Acacia senegal $(1,41 \%)$ et Balanites aegyptiaca $(1,17 \%)$ qui présentent un recouvrement important tandis que les espèces Boscia sengalensis $(0,655 \%)$, Capparis decidua $(0,452 \%)$, Maerua crassifolia $(0,80 \%)$ et Zizyphus mauritiana $(0,37 \%)$ présentent un recouvrement relativement important.

\section{Surface terrière}

La zone d'étude présente une surface terrière de 2,107 $\mathrm{m}^{2} \cdot$ ha $^{-1}$. L'examen de ce paramètre donne les résultats suivant pour les différents sites : Wadi Fira Est 2,07 $\mathrm{m}^{2} \cdot \mathrm{ha}^{-1} \pm$ 0,03 et Ennedi Est $2,15 \mathrm{~m}^{2} \cdot \mathrm{ha}^{-1} \pm 0,02$. Les différentes espèces que présentent les surfaces terrières les plus importantes dans les deux différents sites sont les suivantes :

Au Wadi Fira Est: Acacia raddiana $\left(0,636 \mathrm{~m}^{2} \cdot \mathrm{ha}^{-1}\right)$, Balanites aegyptiaca $(0,60$ $\left.\mathrm{m}^{2} \cdot \mathrm{ha}^{-1}\right)$, Acacia senegal $\left(0,324 \mathrm{~m}^{2} \cdot \mathrm{ha}^{-1}\right)$, Faidherbia albida $\left(0,239 \mathrm{~m}^{2} \cdot \mathrm{ha}^{-1}\right)$ et Capparis decidua $\left(0,12 \mathrm{~m}^{2} \cdot \mathrm{ha}^{-1}\right)$. Les espèces Boscia senegalensis $\quad\left(0,09 \quad \mathrm{~m}^{2} \cdot \mathrm{ha}^{-1}\right), \quad$ Zizyphus mauritiana $\left(0,05 \quad \mathrm{~m}^{2} \cdot \mathrm{ha}^{-1}\right)$ et Maerua crassifolia $\left(0,01 \mathrm{~m}^{2} \cdot \mathrm{ha}^{-1}\right)$ présentent les surfaces terrières les moins importantes.
A l'Ennedi Est: Acacia raddiana $\left(1,188 \mathrm{~m}^{2} \cdot \mathrm{ha}^{-1}\right)$, Maerua crassifolia $(0,28$ $\left.\mathrm{m}^{2} \cdot \mathrm{ha}^{-1}\right)$, Boscia senegalensis $\left(0,24 \mathrm{~m}^{2} \cdot \mathrm{ha}^{-1}\right)$, Balanites aegyptiaca $\left(0,20 \mathrm{~m}^{2} \cdot \mathrm{ha}^{-1}\right)$, Acacia senegal $\left(0,12 \mathrm{~m}^{2} \cdot \mathrm{ha}^{-1}\right)$ et Capparis decidua $\left(0,11 \mathrm{~m}^{2} \cdot \mathrm{ha}^{-1}\right)$. L'espèce Zizyphus mauritiana $\left(0,012 \mathrm{~m}^{2} \cdot \mathrm{ha}^{-1}\right)$, la moins importante en surface terrière dans ce site.

\section{Régénération naturelle du peuplement ligneux}

La régénération naturelle du peuplement ligneux a été évaluée par l'importance des jeunes plants recensés : 921 jeunes plants, soit un taux de régénération naturelle de $72,20 \%$ pour l'ensemble de la zone étudiée. Cette régénération naturelle de 422 individus dans le Wadi Fira Est soit un taux de $63,46 \%$, mais elle est relativement faible dans l'Ennedi Est avec 243 individus, soit 36,54\% de taux de régénération naturelle (Figure.6). Dans les deux sites étudiés, Acacia raddiana régénère plus avec un taux de régénération naturelle de 40,17\%, suivie de Boscia senegalensis avec $14,44 \%$. Ces deux espèces dominantes ont donc les taux de régénération naturelle les plus élevés $(54,61 \%)$ du taux global de régénération naturelle dans les deux sites.

\section{Anthropisation du peuplement ligneux}

Des calculs ont été effectués au sein du peuplement ligneux dans les deux sites en vue d'apprécier l'état d'anthropisation du couvert ligneux de la zone d'étude. Une fréquence de $10,94 \%$ de pieds morts ou abattus dans toute la zone d'étude a été notée. Dans le Wadi Fira Est où le taux d'anthropisation est plus important $(16,81 \%)$, les individus abattus ou morts sur pieds, ont été tous des pieds d'Acacia raddiana (Figure.7).

Dans l'Ennedi Est où le taux d'anthropisation moins important $(5,84 \%)$, les individus morts sur pieds ont été des Acacia raddiana. La plupart des placettes n'ont pas fait l'objet d'une anthropisation du peuplement ligneux. Ainsi, à travers l'ensemble des deux sites, les placettes ne présentaient pas des traces anciennes ou récentes de feux de brousse. 
Distribution des variables morphométriques des ligneux pour chaque site

La distribution des variables morphométriques des ligneux est représentée par des histogrammes (Figures. 8 et 9). L'étude statistique a porté sur la des circonférences à 30 $\mathrm{cm}$ et de la hauteur totale.

\section{Distribution de la circonférence à $30 \mathrm{~cm}$}

La distribution des individus par classe de circonférence dans la zone d'étude est illustrée par la Figure 8. Les arbres de petite circonférence représentaient 53\% (classes 1, 2 et 3 cumulées) de la population totale de la zone d'étude. Par contre, les individus constituant les classes de circonférence supérieure à $120 \mathrm{~cm}$ représentent moins de $10 \%$. L'analyse de la structure en circonférence des ligneux présente une allure en «J renversé » et s'ajustait à la distribution théorique de Weibull avec le paramètre de forme $\mathrm{c}=1,837$ $(1<\mathrm{C}<3,6)$, caractéristique des peuplements avec une prédominance des individus jeunes ou de petites circonférence. Cette même tendance est observée dans la zone de Wadi Fira Est, les individus de circonférence compris entre 15 et $60 \mathrm{~cm}$ étaient les plus abondants soit plus de 45\%; ceux de circonférence supérieure à 120 cm sont moins de $5 \%$.

Par contre dans la province de l'Ennedi Est, même si la distribution asymétrique droite (Weibull avec un paramètre de forme $\mathrm{c}=2,145$ $(1<\mathrm{C}<3,6)$, il y a une présence assez importante d'individus de circonférence supérieure à $120 \mathrm{~cm}(+10 \%)$.

\section{Distribution de la hauteur}

A l'échelle du peuplement, les hauteurs des individus varient de 1 à $16 \mathrm{~m}$. Dans les différentes zones, une distribution asymétrique droite avec prédominance d'individus ayant moins de $4 \mathrm{~m}$ de hauteur a été observée sur l'étendue de la zone d'étude ( $+45 \%)$. C'est une zone à peuplement arbustif dominant. Cette même tendance observée dans les différentes régions du tracé de la GMV.

Tableau 1 : Liste des espèces inventoriées dans la zone d'étude.

\begin{tabular}{|c|c|c|c|c|c|}
\hline \multicolumn{4}{|c|}{ TAXONS } & \multicolumn{2}{|c|}{ SITES } \\
\hline Familles & Genres & Espèces & Code & $\mathbf{1}$ & 2 \\
\hline BALANITACEAE & Balanites & Balanites aegyptiaca (L.) Del. & $B a a$ & + & + \\
\hline \multirow{3}{*}{ CAPPARIDACEAE } & Capparis & Capparis decidua (Forssk) Edgew & $\mathrm{Cad}$ & + & + \\
\hline & Boscia & Boscia senegalensis (Pers.) & Bos & + & + \\
\hline & мaеrua & Maerua crassifolia Forssk & Mac & + & + \\
\hline \multirow{3}{*}{ MIMOSACEAE } & & Acacia senegal $(L$, ) Willd & Acs & + & + \\
\hline & Acacia & Faidherbia albida (Del.) A. Chev. & Faa & + & \\
\hline & & Acacia raddiana (Savi) Brenan & Acr & + & + \\
\hline RHAMNACEAE & Zizyphus & Zizyphus mauritiana Lam. & Zim & + & + \\
\hline 4 familles & 6 genres & 8 espèces & & 8 & 7 \\
\hline
\end{tabular}

Tableau 2 : Diversité floristique des différentes zones.

\begin{tabular}{lccc}
\hline Paramètres de diversité & ZE & Wadi Fira Est & Ennedi Est \\
\hline Richesse spécifique $(\mathrm{S})$ & 8 & 8 & 7 \\
\hline Indice de Shannon $\left(\mathrm{H}^{\prime}\right)$ & 2,53 & 2,45 & 2,71 \\
\hline Equitabilité de Pielou (E) & 0,84 & 0,82 & 0,90 \\
\hline
\end{tabular}


Tableau 3 : Paramètres structuraux de la végétation ligneuse de la zone d'étude.

\begin{tabular}{lccc}
\hline Unité topographique & Zone & Wadi Fira Est & Ennedi Est \\
\hline Effectif (individus) & 257 & 119 & 138 \\
\hline Surface (ha) & 6,75 & 3,25 & 3,5 \\
\hline Dm entre arbres (m) & 11,69 & 10,73 & 12,52 \\
\hline Densité théorique (ind/ha) & 73,18 & 86,86 & 63,80 \\
\hline Densité réelle (ind/ha) & $38,07 \pm 11,8$ & $36,62 \pm 4,24$ & $39,43 \pm 5,4$ \\
\hline Rapport Dth/Dob & 1,92 & 2,37 & 1,62 \\
\hline Surface terrière (m/ha) & $2,11 \pm$ & $2,07 \pm 0,03$ & $2,15 \pm 0,02$ \\
\hline Taux de recouvrement aérien (\%) & 17,82 & 18,18 & 17,49 \\
\hline
\end{tabular}

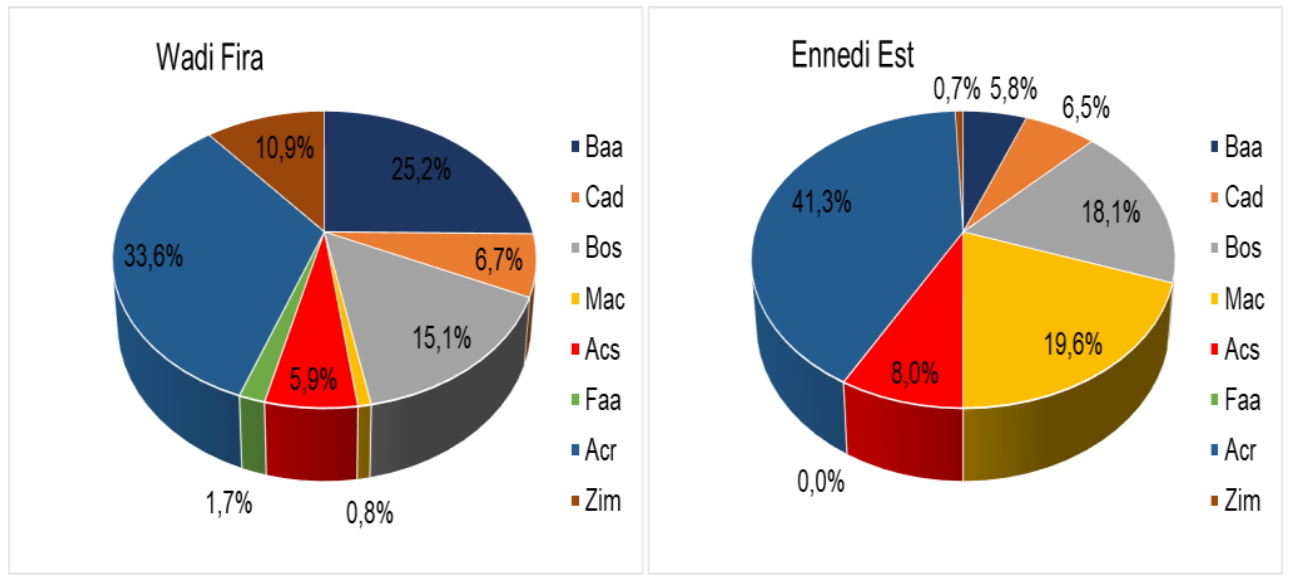

Figure 5 : La fréquence spécifique.

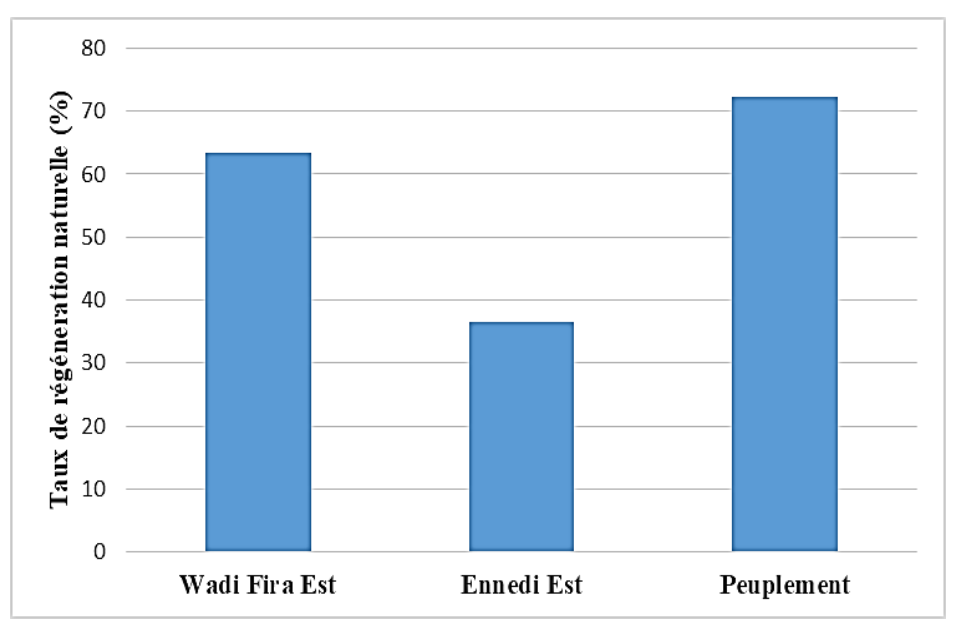

Figure 6 : Taux de régénérations naturelles. 
M. MBAIKOUBOU et al. / Int. J. Biol. Chem. Sci. 15(5): 1885-1901, 2021

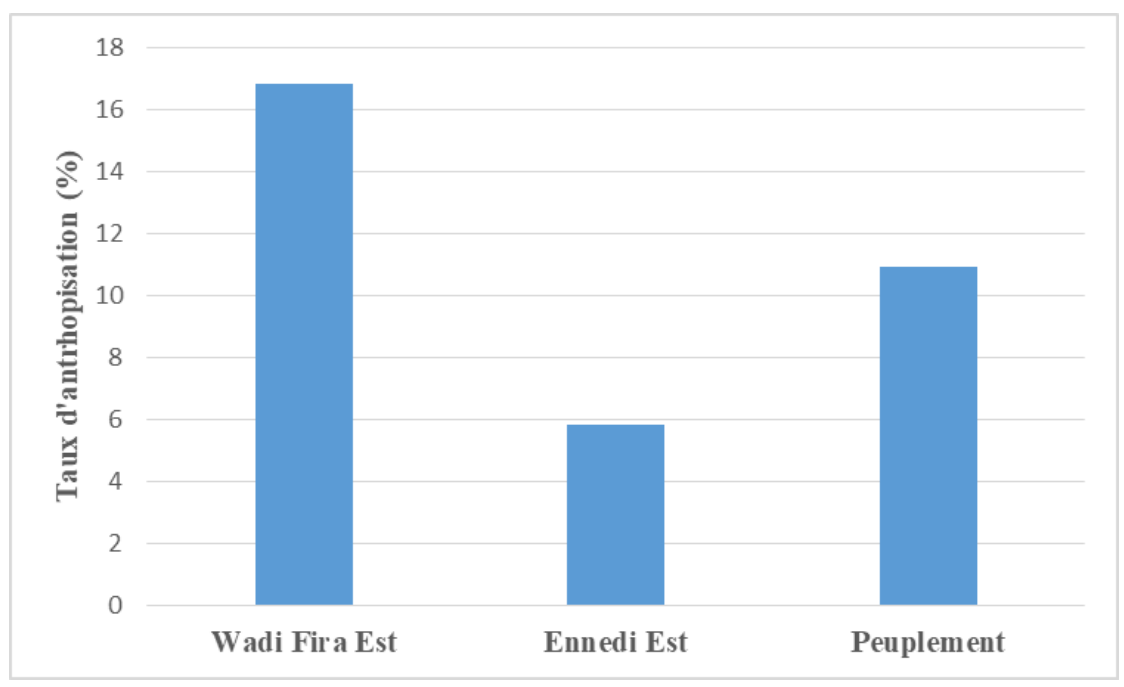

Figure 7 : Taux d'anthropisation (A. raddiana).

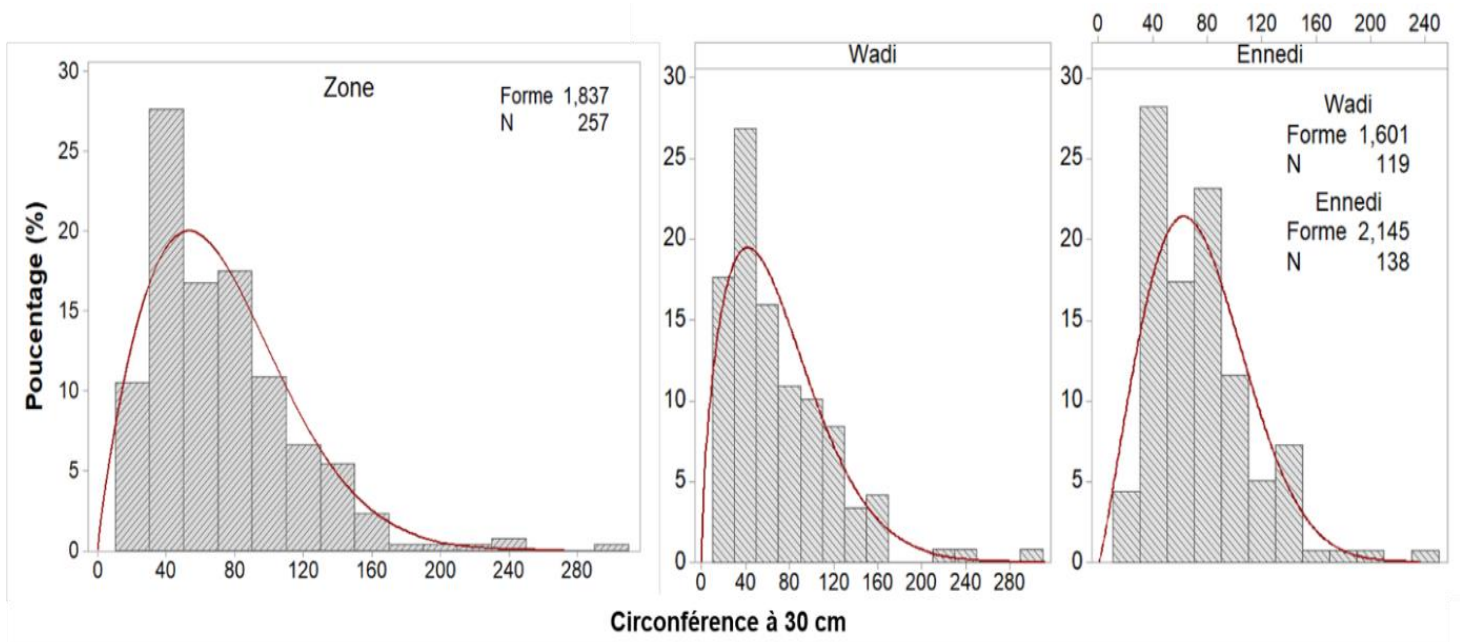

Figure 8 : Histogramme des circonférences à $30 \mathrm{~cm}$.
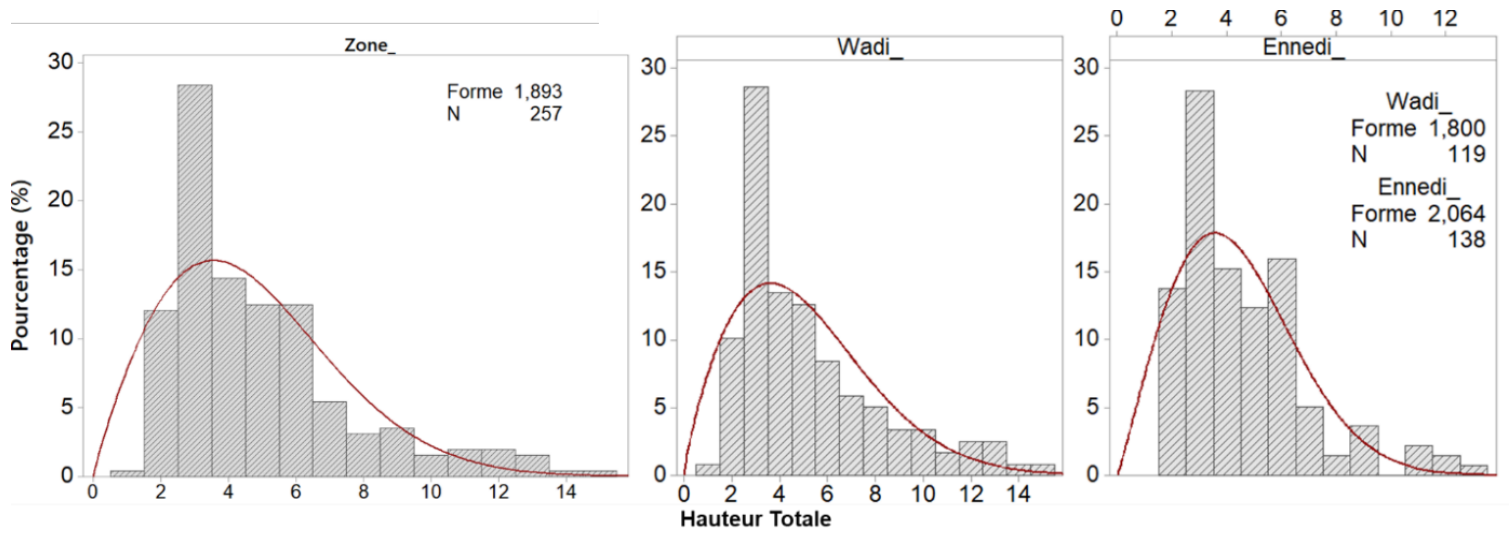

Figure 9: Histogramme des hauteurs totales. 


\section{DISCUSSION}

La caractérisation du peuplement ligneux de la zone d'étude a révélé 254 individus répartis dans 8 espèces; ce qui démontre à suffisance le caractère de l'extrême pauvreté de la flore, en lien avec la superficie de la zone d'étude. Les études menées par Minda (2015) dans la zone sahélienne du Tchad (Lac, Barh El gazal et Kanem) et Niang (2009, 2014) dans la zone sahélienne du Sénégal ont donné des résultats similaires. Mais les présents résultats diffèrent de ceux obtenus par Boubacar (2010) en zone soudanienne et sahélo-saharienne du Niger et par Goy (2012) en zone sahélo-soudanienne du Tchad, où ils ont obtenu respectivement 24 espèces et 44 espèces. La diversité et la richesse floristique sont inférieures à celles des formations végétales des zones sahéliennes du Burkina Faso (54 espèces, 19 familles) décrites par Sawadogo et al. (2016) et Mahamat et al. (2021) en zone sahélienne du Tchad. Cette faible diversité et richesse floristique corrobore à ce qu'ont affirmé Ouedraogo et al. (2006) que les zones soudaniennes sont plus riches que les zones sahéliennes ayant que des espèces spécifiques.

La présence massive de $B$. aegyptiaca après l'Acacia $s p$ s'explique par une prolifération de $B$. aegyptiaca dans les végétations pâturées dans le contexte de fortes pressions pastorales comme le souligne Béchir et Mopaté (2015) dans le Batha Ouest au Tchad et Chanceyambaye et al. (2017). En effet, la richesse floristique reste un indicateur indiquant la répartition et par comparaison dans le temps, sur l'évolution des espèces dans une zone bien donnée. Il est apparu clairement à la lumière de ces différents résultats obtenus par ces auteurs que la zone de la présente étude une diversité floristique très faible.

Les espèces communes aux deux sites au nombre de sept (07), sont : Balanites aegyptiaca, Capparis decidua, Maerua crassifolia, Boscia senegalensis, Zizyphus mauritiana, Acacia senegal et Acacia raddiana. L'espèce Faidherbia albida n'est rencontrée que dans le Wadi Fira Est. Cette faible phytodiversité se justifie probablement par de longues années de sécheresse et par l'impact des activités humaines. Les espèces les plus abondantes des écosystèmes de la zone d'étude sont Acacia raddiana, Boscia sengalensis, Balanites aegyptiaca et Maerua crassifolia. Ceci traduit une certaine stabilité et donc une résistance de ces espèces aux aléas climatiques (Bakhoum, 2013). L'espèce Acacia raddiana est présente dans toute la zone d'étude avec un nombre très important d'individus. Dans la zone d'étude, elle se développe sur les sols sablo-argileux.

D'après les études réalisées par les auteurs (Mahamat et al., 2021), Acacia raddiana contribue de manière significative à la diminution du phénomène de désertification dans les zones arides et sahariennes. Et bien plus, A. raddiana est présente dans toutes les régions arides et semi-arides, allant du Sénégal au centre de l'Afrique, du Tchad jusqu'à Djibouti où il constitue la limite des arbres dans le désert et serait sans nul doute, l'arbre le plus abondant au Tchad d'après (Mahamat et al., 2012). Son système racinaire est très développé et très résistant à la sécheresse ; ceci pourrait permettre de consolider les dunes et de stopper le mouvement des sables. Elle constitue avec Balanites aegyptiaca les espèces les plus abondantes dans les sites de cette étude. La sécheresse des années 1970 a provoqué une concentration de la végétation ligneuse et parfois une nette dominance de certaines espèces comme Balanites aegyptiaca (Diatta, 2008), ce qui est en accord avec les présents résultats. Balanites aegyptiaca de la famille des Balanitaceae (Arbonnier, 2000) constitue la deuxième espèce qui domine dans cette zone après A. raddiana.

Partout, la densité réelle est plus faible que la densité théorique. En effet, le rapport entre la densité théorique et la densité réelle varie de 2,087 dans le Wadi Fira Est et de 1,618 dans l'Ennedi Est. Le rapport entre la densité 
théorique et la densité réelle est plus élevé dans le Wadi Fira Est, ce qui traduit une distribution en agrégats d'individus. Par contre dans l'Ennedi Est. Ce rapport est faible et cela explique une distribution régulière (Canard et Poinsot, 2004) et (Minda et al., 2015). La surface terrière est plus élevée à Wadi Fira Est mais elle l'est moins dans l'Ennedi Est. En effet, l'importance de cette surface terrière déterminée dans le Wadi Fira Est pourrait s'expliquer en effet, par la forte densité de certaines espèces (Acacia raddiana et Balanites aegyptiaca) et par la présence des arbres à gros troncs (Faidherbia albida, etc.). Cependant, la faible surface terrière constatée dans l'Ennedi pourrait être liée au fait que cette zone est mise en profit pour les pâturages des troupeaux et des prélèvements de bois à usage divers (Ndiaye et al., 2014 ; Niang et al., 2014). La surface terrière faible est également caractérisée par la dominance d'arbustes (Boscia senegalensis).

Le taux de recouvrement est faible et à Wadi Fira Est et à l'Ennedi Est. Cette baisse drastique du taux de recouvrement observé pourrait être expliquée par l'ampleur des activités anthropiques (abattage des arbres et élevage), (Minda et al., 2013). La forte régénération des $A$. raddiana, $B$. senegalensis et $Z$. mauritiana s'expliquerait par leur capacité d'émettre des rejets suite au défrichage lors de l'installation des cultures. Les mêmes observations ont été faites par Massaoudou Moussa et al. (2015) sur la régénération des sites restaurés au Niger.

La présente étude a révélé que la plupart des espèces qui poussent dans la zone de l'étude et les zones semblables sont des épineux. Ces résultats corroborent bien avec ceux rapportés par Daniel et Hubert (1991).

\section{Conclusion}

Le présent travail a contribué à mettre en évidence le potentiel de régénération des peuplements ligneux du tracé de la Grande Muraille Verte du Tchad précisément dans sa partie de Wadi Fira Est et de l'Ennedi Est. Présentes de façon massive, les espèces végétales Acacia raddiana et Balanites aegyptiaca ont un taux de renouvellement très important, attestant de leur adaptation aux conditions pédoclimatiques et aux actions anthropiques dans cette zone; En raison de cela, elles doivent être privilégiées dans les programmes de restauration de ces écosystèmes. Ainsi une exploration supplémentaire des espèces végétales constitue un volet important pour la conception de stratégies de mise en valeur des sites du tracé de la GMV du Tchad. Ces investigations ultérieures à mener peuvent apporter une réponse adaptative dans la lutte contre la désertification.

\section{CONFLIT D'INTERETS}

Les auteurs déclarent qu'ils n'ont aucun conflit d'intérêts entre eux par rapport à cet article.

\section{CONTRIBUTIONS DES AUTEURS}

$\mathrm{MM}$ a assuré la conduite de travaux sur le terrain et la rédaction de la première version du manuscrit. TD a contribué à la collecte des données sur le terrain. AG, AD, NF et MMS ont contribué à la correction et à l'amélioration du manuscrit. AG a encadré et supervisé le travail.

\section{REMERCIEMENTS}

Ce travail a été réalisé grâce à l'appui financier de l'Institut Tchadien de Recherche Agronomique pour le Développement (ITRAD). Nous adressons notre reconnaissance à l'ITRAD à travers les responsables à savoir le Directeur Général (DG) et le Directeur Scientifique (DS). Les auteurs témoignent également leur gratitude aux collègues des services déconcentrés de l'Etat de la zone de l'étude qui leur ont facilité le travail pratique sur le terrain lors des missions de collecte des données. 


\section{REFERENCES}

ANAM (Agence Nationale pour la Météorologie au Tchad). 2020. Paramètres météorologiques relatifs à la période 1987-2017 N'Djamena-Tchad. Fiche de relevés climatiques-ANAM, 15 p.

Arbonnier M. 2000. Arbres, Arbustes et Lianes des Zones Sèches d'Afrique de l'Ouest. CIRAD, Paris.

ASECNA (Agence pour la Sécurité de la Navigation Aérienne en Afrique et à Madagascar). 2012. Paramètres météorologiques relatifs à la période 2011-2012. Fiche de relevés climatiquesASECNA, Ndjamena-Tchad, $12 \mathrm{p}$.

Ba M, Bourgoin J, Thiaw I, Soti V. 2018. Impact des modes de gestion des parcs arborés sur la dynamique des paysages agricoles, un cas d'étude au Sénégal. $L a$ Revue Electronique en Sciences de l'Environnement VertigO, 18(2). https://id.erudit.org/iderudit/1059927ar

Bakhoum A. 2013. Dynamique des ressources fourragères : indicateur de résilience des parcours communautaires de Téssékéré au Ferlo (Nord-Sénégal). Thèse de Doctorat unique, FST-UCAD, 115p.

Béchir AB, Mopate LY. 2015. Analyse de la dynamique des pâturages autour des ouvrages hydrauliques des zones pastorales du Batha Ouest au Tchad. Afrique Science, 11(1): 212-226. http://www.afriquescience.info.

Boubacar H. 2010. Caractérisation biophysique des ressources ligneuses dans les zones dégradées et reverdies au Sahel : cas du département de Mayahi. Option «Protection et Restauration des Ecosystèmes Soudaniens et SahéloSahariens». Mémoire de DEA, Université Abdou Moumouni Niamey, Niger, 69 p.

Canard A, Poinsot D. 2004. Quelques méthodes statistiques typiques de l'étude des populations et des peuplements par la méthode des quadrats. Fiche technique, Université de Renne 1, 34 p.

Chanceyambaye N, Fidèle TN, Ibrahima A. 2017. Caractérisation des ligneux de la savane sahélienne à Acacia senegal (L) Willd dans la région du Guéra, Tchad. International Journal of Applied Research, 3(4) : 600-606.

Dan Guimbo I, Mahamane A, Ambouta K JM. 2010. Peuplement des parcs à Neocarya macrophylla (Sabine) Pranceet à Vitellaria paradoxa (Gaertn. C.F.) dans le sud-ouest nigérien : diversité, structure et régénération. Int. J. Biol. Chem. Sci., 4(5): $\quad 1706-1720 . \quad$ DOI: 10.4314/ijbcs.v4i5.65568

Diatta S. 2008. Modes de propagation d'un ligneux fourrager sahélien : Maerua crassifolia Forsk. Thèse de Doctorat, Université Cheikh Anta Diop, Dakar, 89 p.

Goy S. 2012. Etude phytosociologique et diagnostic faunique du parc national de manda (TCHAD), éléments pour un aménagement. Spécialité : écologie, agroforesterie et adaptations. Mémoire de Master, Université Cheikh Anta Diop, Dakar, $162 \mathrm{p}$.

Grande Muraille Verte. 2009. Colloque scientifique international sur le choix des espèces végétales et des systèmes de mise en valeur et de suivi. Grande Muraille Verte, $47 \mathrm{p}$.

Jalut G, Esteban AA, Bonnet L, Gauquelin T, Fontugne M. 2000. Holocene climatic changes in the Western Mediterranean from south-east France to south-east Spain. Palaeogeography, Palaeoclimatology, Palaeoecology, 160 : 255-290.

Jauffret S. 2001. Validation et comparaison de divers indicateurs des changements à long terme dans les écosystèmes méditerranéens arides, application au suivi de la désertification dans le sud 
tunisien. Thèse d'écologie, Université d'Aix Marseille, Marseille, 328 p.

Kaou Kak, Manzo OL, Dan Guimbo I, Karim S, Habou R, Paul R. 2017. Diversité floristique et structure de la végétation dans la zone dunaire du sud-est du Niger : cas de Mainé Soroa. Journal of Applied Biosciences, 120(1): 14 p. DOI: 10.4314/jab.v120i1.8

Laouali A, Dan Guimbo I, Larwanou M, Inoussa MM, Mahamane A. 2014. Utilisation de Prosopis africana (G. et Perr.) Taub dans le sud du département d'Aguié au Niger : les différentes formes et leur importance. Int. J. Biol. Chem. Sci., 8(3): 1065-1074. DOI: 10.4314/ijbcs.v8i3.20

Larwanou M, Saâdou M, Hamadou S. 2006. Les arbres dans les systèmes agraires en zone sahélienne du Niger : mode de gestion, atouts et contraintes. Tropicultura, 24(1): 14-18.

Magnin F, Martin S. 2004. Evolution climatique, impact anthropique et réponse des peuplements malacologiques holocènes. Proposition d'une méthode d'interprétation. Impact anthropique et évolution climatique de la fin du Néolithique à l'Antiquité. Actes du colloque de Carcassonne, novembre 2000, 64-71.

Mahamat SM, Temoua D, Goy S, Goalbaye T. 2012. Compte rendu de la mission de terrain relatif aux travaux de recherche doctorale sur le tracé de la Grande Muraille Verte au Tchad. Grande Muraille Verte, Tchad, $18 \mathrm{p}$.

Mahamat AG, Diallo MD, Diallo A. Minda MS, Guisse A. 2021. Distribution des ligneux sur le tracé de la grande muraille verte : cas de batha et de wadi-fira Ouest au Tchad. Int. J. Biol. Chem. Sci., 15(1): 144-155.

DOI: https://dx.doi.org/10.4314/ijbcs.v15i1.1

Massaoudou M, Larwanou M, Mahamane S. 2015. Caractérisation des peuplements ligneux des parcs à Faidherbia albida (Del) A. Chev. et à Prosopis africana (Guill., Perrot et Rich.) Taub. du CentreSud Nigérien. Journal of Applied Biosciences, 94:8890 - 8906. DOI: 10.4314/jab.v94i1.6

Minda MS, Diallo A, Ndiaye O, Madiara NF, Guissé A. 2013. Caractérisation des peuplements ligneux de la zone Cayor Baol (Thiès Sénégal). Int. J. Biol. Chem. Sci., 7(5): 2117-2132. DOI: 10.4314/ijbcs.v7i5.28

Minda M. 2015. Caractérisation des sols et de la végétation ligneuse sur le tracé de la grande muraille verte du Tchad. Thèse de Doctorat, Département de Biologie Végétale, UCAD-FST, 148 p.

Moussa M, Larwanou M, Mahamane S. 2015. Caractérisation des peuplements ligneux des parcs à Faidherbia albida (Del) A. Chev. et à Prosopis africana (Guill., Perrot et Rich.) Taub. du Centre-Sud Nigérien. Journal of Applied Biosciences, 94(1):8890. DOI: 10.4314/jab.v94i1.6

Ndiaye O, Diallo A, Stephen AW, Guissé A. 2014. Structural Diversity of Woody Species in the Senegalese Semi-Arid Zone-Ferlo. American Journal of Plant Sciences, 5: 416-426. DOI: http://dx.doi.org/10.4236/ajps.2014.5305 5

Ngom D, Boubacar C, Sagna B, Gomis ZD. 2018. Cortège floristique, paramètres structuraux et indicateurs d'anthropisation des parcs agroforestiers à Elaesis guineensis Jacq. en basse Casamance, Sénégal. Journal of Animal and Plant Sciences, 36(3): 5919-5932.

Niang K. 2009. L'arbre dans les parcours communautaires du Ferlo-Nord (Sénégal). Mémoire DEA, FST, UCAD, Sénégal, 69 p.

Niang K. 2014. Caractérisation de la végétation ligneuse du Ferlo et son usage par les 
populations locales. Mémoire de Master, FST, UCAD, Sénégal, 133 p.

Niang K, Ndiaye O, Diallo A, Guisse A. 2014. Flore et structure de la végétation ligneuse le long de la Grande Muraille Verte au Ferlo, nord Sénégal. Journal of Applied Biosciences, 79: 6938-6946. DOI: $10.4314 /$ jab.v79i1.15

Ouédraogo A, Thiombiano A, Hahn-Hadjali K, Guinko S. 2006. Diagnostic de l'état de dégradation des peuplements de quatre espèces ligneuses en zone soudanienne du Burkina Faso. Sc. Chang. Plan./Séch., 17: 485-491. DOI: 10.1684/SEC.2006.0058

Pias J. 1970. La végétation du Tchad : ses rapports avec les sols, variations paléobotaniques au quaternaire. Contribution à la connaissance du Bassin tchadien. ORSTOM, Tchad, 49 p.

Piot J, Diaite A. 1983. Système de production d'élevage au Sénégal : étude du couvert ligneux. Compte rendu de fin d'étude, C.I.T., Nogent sur Marne, ISRA, DakarHann, $30 \mathrm{p}$.

Sall P. 1996. Les parcs agro-forestiers du Sénégal : Etat des connaissances et perspectives. Rapport de consultation SALWA, $144 \mathrm{p}$.

Salvador PG, Vérot-Bourrély A, Bravard JP, Franc O, Macé S. 2002. Les crues du Rhône à l'époque gallo-romaine dans la région lyonnaise: les fleuves ont une histoire. In Paléo-Environnement des Rivières et des Lacs Français Depuis 15 000 Ans, Bravard JP, Magny M (Eds). Errance : Paris ; 215-221.
Sarr O, Diatta AS, Gueye M, Ndiaye PM, Guisse A, Akpo LE. 2013. Importance des ligneux fourragers dans un système agropastoral au Sénégal (Afrique de l'Ouest). Revue Méd. Vét., 164 (1) : 2-8.

Sawadogo OM, Ouattara K, Pare S, Ouedraogo I, Sawadogo-Kaboré S. 2016. Structure, composition spécifique et diversité des ligneux dans deux zones contrastées en zone Sahélienne du Burkina Faso. Revue Electronique en Sciences de l'Environnement, $\quad \mathbf{1 6}(1)$ DOI: https://doi.org/10.4000/vertigo.17282

Van Geel B, Magny M. 2002. Mise en évidence d'un forçage solaire du climat à partir de données paléo écologiques et archéologiques : la transition SubboréalSubatlantique. In Equilibres et Ruptures dans les Ecosystèmes Depuis 20000 ans en Europe de l'Ouest, Richard H, Vignot A (Eds). Presses Universitaires FrancComtoises: Besançon ; 107-122.

Wouters P, Notelaers V. 1999. L'espace entre les arbres et la densité des peuplements. Sylva Belgica, 106 (21): 4.

Yaya Issifou Moumouni, Ismaïla Toko Imorou, Abdel Djalilou Moussa. 2019. Caractérisation de la Dégradation à Travers la Diversité Floristique et la Structure de la Végétation dans le Bassin Moyen de la Sota au Nord-Bénin. International Journals of Sciences and High Technologies, 18(1): 53-70.

Young A. 1995. Agroforesterie pour la conservation des sols. ICRAF, Tchad, $183 \mathrm{p}$. 